\title{
Is preventive suicide a rational response to a presymptomatic diagnosis of dementia?
}

It may soon be possible to diagnose neurodegenerative disorders, such as early onset Alzheimer's disease, with a high degree of accuracy well before these conditions become symptomatic. In a carefully argued and thought-provoking piece, Dena Davis (see page 543, Editor's choice) maintains that preemptive suicide may be a rational option for those confronted with a preclinical diagnosis of impending dementia, and consequently that withholding the results of dementia research until effective treatments become available constitutes an unjustified infringement on patient autonomy. If suicide is indeed a rational course of action for some people diagnosed with dementia, then contrary to received wisdom, biomarker information indicating a high risk of dementia may be 'actionable' even if there is no known treatment for the condition. Preemptive suicide, Davis argues, may be motivated and justified by a mixture of other-regarding and self-regarding considerations. These include, inter alia, the wish to avoid imposing significant financial or psychological hardships on one's family, the unwillingness to live without the capacities for meaningful independence or agency, and the desire to write a final chapter that is consistent with one's stable values and that does not distort or eclipse the overarching narrative of one's life.

Dementia raises an ethical dilemma that does not arise in connection with other diseases with respect to which many believe suicide is a rational course of action (such as terminal cancer). This dilemma arises from the fact that a person with impending dementia cannot wait until the disease takes hold to issue and carry out a decision to end his or her life, since dementia destroys the capacities that underpin rational decision-making. Because jurisdictions permitting suicide require, quite reasonably, that a patient be shown to have rational decision-making capacities, it is impossible for patients with later stages of dementia, which significantly attenuates those capacities, to legally commit suicide. And while advanced directives could in theory offer a way out of this dilemma, past decisions made by the pre-demented individual are often taken to have weaker moral force than the contemporary choices or preferences of the post-demented individual. In addition, unlike many other terminal illnesses, dementia may implicate philosophical conceptions of identity that are of great significance to these ethical questions. For instance, if the pre-demented individual and the post-demented individual are sufficiently psychologically discontinuous so as to constitute different persons, then this undermines appeals to the autonomy of the pre-demented patient as a controlling or even weighty ethical variable in such cases. Many would hold that the degree of psychological discontinuity necessary to result in two different living persons is not met in cases of dementia, or that the severely demented life does not amount to the life of a person and hence that there is no actual conflict of interests. But the looming prospect of identity annihilation, to the extent that it exists, may give pre-demented persons further weighty reasons to commit preemptive suicide. For it may cause individuals, in Davis's words, to view "a diagnosis of impending dementia [as] a warning that one is about to be invaded by an enemy army that will always win. [In such cases, it] is entirely sensible to burn down the fort and refuse it a home."

Presymptomatic diagnoses of most types of dementia make only probabilistic predictions of developing the condition, and such predictions are unlikely to asymptotically approach certainty anytime soon. Rebecca Dresser (see page 550), in her commentary on Davis, argues that the probabilistic nature of presymptomatic diagnoses undermines the rationality of suicide, because it suggests that such diagnoses will be rife with false positives, and because the optimal moment for ending one's life will remain elusive. I take Dresser to be using the phrase 'false positive' here in a non-standard sense to refer to the false belief that dementia will inevitably manifest when its occurrence is in fact uncertain-rather than to a test result which finds the presence of a biomarker indicating a high risk of dementia when in fact that biomarker is absent. If false positives in relation to the presence of the relevant biomarkers were indeed rife, this would raise different and very serious ethical concerns that cut further against the rationality of preemptive suicide as a response to preclinical diagnosis. Such a scenario seems unlikely, however, given that the false positive rates associated with genetic testing are much lower (due to the technical reliability of genetic assays) than they are for other preventive screens, such as imaging to detect cancer.

It follows that one must balance the risk that preemptive suicide will end one's life earlier than necessary against the risk that one will develop dementia and become stripped of rational decision-making capacities before one is able to act. But this is no different, Davis argues, than the riskbalancing that patients are routinely engaged in when faced with presymptomatic diagnoses of non-neurodegenerative diseases that do not exhibit $100 \%$ penetrance-as is the case for patients contemplating whether and when to have a preventive mastectomy in light of a positive BRCA-1 result. Of course, one may query whether this analogy holds, given that preemptively taking one's own life is very different from choosing to have a preventive mastectomy. Nevertheless, like presymptomatic analyses, diagnoses of dementia based on early ambiguous symptoms also make probabilistic predictions about the underlying cause as well as the nature and timing of the projected outcome. The crux of the issue, therefore, turns not on whether symptoms have in fact manifested, but on how likely and subjectively harmful the projected outcome must be in order to warrant preemptive action. Davis seems to believe that the answer to this question will vary from person to person, depending on how risk-averse one is and how one balances the various values and interests at stake in the decision. Dresser, on the other hand, seems to believe that preemptive suicide is only justified, if ever, when very high probabilities of developing dementia are appropriately assigned.

In the interests of clarification, we might modify slightly the terminology of the debate. In legal philosophy, preemption 
describes a response to a threat that is 'imminent' (instant and overwhelming), whereas prevention entails prophylactic actions taken sometime prior to a threat's becoming imminent. Harms that are subject to prevention have, ceteris paribus, a lower subjective probability of occurrence than harms that are subject to preemption, due to the larger temporal gap between prevention and the contemplated harm, during which time intervening causes and additional relevant information could lower the probability calculation. Insofar as the justification for an intervention hinges (in part) on the high probability of the outcome that one seeks to avoid, prevention will, ceteris paribus, tend to be on weaker justificatory grounds than preemption. In the present context, however, we should not assume that preclinical indicators of neurodegenerative disease will always admit of weaker predictions than diagnoses based on early symptoms. It remains an open empirical question whether early phenotypic symptoms 'screen-off' genetic biomarkers with respect to the probability of developing a given disease; in point of fact, it seems more likely that both symptoms and biomarkers will be used to inform the probability calculus. The key philosophical question is whether preventive suicide is ever a rational response to learning that one has a certain risk of developing dementia, regardless of whether the diagnosis is predicated on symptoms or presymptomatic biomarkers or both. Davis argues that preventive suicide may be rational in cases where preemptive suicide will be thwarted due to progression of the disease, whereas Dresser argues that suicide in the face of impending dementia is always irrational except, perhaps, in genuine cases of preemption.
Dresser stresses that clinicians should ensure that patients with presymptomatic diagnoses of dementia understand that their probability of developing the condition may be significantly less than $100 \%$, and that there is a full range of care options for people living with the disease. Davis would presumably agree with these remarks, while maintaining that healthcare practitioners should not operate on the patronizing and autonomy-diminishing assumption that there is no morally permissible and efficacious course of action for patients at high risk of dementia. These ethical questions will come increasingly to the fore as we hone our ability to predict neurodegenerative disorders before any symptoms are manifest-compelling us to reexamine the circumstances in which suicide is a reasonable response to disease, and to consider the legal and policy implications of our conclusions. 